Article

\title{
The Reaction of Ethyl 2-oxo-2H-chromene-3-carboxylate with Hydrazine Hydrate
}

\author{
Hatem A. Abdel-Aziz ${ }^{1,2, *}$, Tilal Elsaman ${ }^{1}$, Mohamed I. Attia ${ }^{1,3}$ and Amer M. Alanazi ${ }^{1}$ \\ 1 Department of Pharmaceutical Chemistry, College of Pharmacy, King Saud University, \\ P.O. Box 2457, Riyadh 11451, Saudi Arabia \\ 2 Department of Applied Organic Chemistry, National Research Center, Dokki, Cairo 12622, Egypt \\ 3 Department of Medicinal and Pharmaceutical Chemistry, National Research Center, Dokki, \\ Cairo 12622, Egypt \\ * Author to whom correspondence should be addressed; E-Mail: hatem_741@yahoo.com; \\ Tel.: +966-1467-3764; Fax: +966-1467-6220.
}

Received: 12 January 2013; in revised form: 29 January 2013 / Accepted: 30 January 2013 / Published: 6 February 2013

\begin{abstract}
Although salicylaldehyde azine (3) was reported in 1985 as the single product of the reaction of ethyl 2-oxo- $2 \mathrm{H}$-chromene-3-carboxylate (1) with hydrazine hydrate, we identified another main reaction product, besides $\mathbf{3}$, which was identified as malono-hydrazide (4). In the last two decades, however, some articles have claimed that this reaction afforded exclusively hydrazide $\mathbf{2}$ and they have reported the use of this hydrazide $\mathbf{2}$ as a precursor in the syntheses of several heterocyclic compounds and hydrazones 6. We reported herein a study of the formation of $\mathbf{2}$ and a facile route for the synthesis of the target compounds $N^{\prime}$-arylidene-2-oxo- $2 H$-chromene-3-carbohydrazides $\mathbf{6 a}-\mathbf{f}$.
\end{abstract}

Keywords: salicylaldehyde azine; malonohydrazide; coumarins; hydrazides/hydrazones; ring-opening

\section{Introduction}

The hydrazides are very useful starting materials for the construction of several functionalized heterocycles with a broad spectrum of biological activities, and consequently they have been studied in considerable detail over the decades [1,2]. For instance, hydrazides are versatile raw materials to synthesize pyrroles [3], pyrazoles [4], 1,3-thiazoles [5], 1,3,4-oxadiazoles [6], 1,2,4-triazoles [7], 1,3,4- 
thiadiazoles [8], 1,2,4-triazolo[3,4-b]-1,3,4-thiadiazoles [9] and 1,2,4-triazolo[3,4-b]-1,3,4-thiadiazines [10]. The common practical route for hydrazide synthesis is the treatment of esters with hydrazine hydrate. The use of microwave irradiation is a recent contribution to this field for the facile preparation of hydrazides by solvent-free reactions of acid derivatives with hydrazine hydrate [11,12]. On the other hand, there is a great deal of interest in chromene-2-ones, especially 3-substituted derivatives, due to their important pharmacological effects, including analgesic, anti-arthritis, anti-inflammatory, anti-pyretic, anti-viral, anti-cancer and anticoagulant properties [13-15]. In the light of previous data and in continuation of our interest in the chemistry of hydrazides [16-24], our efforts targeted the synthesis of hydrazide 2 through the title reaction according to the literature methods [25-36], with the intention of subsequently treating hydrazide $\mathbf{2}$ with different aldehydes to obtain hydrazones $\mathbf{6 a}-\mathbf{f}$ for our biological screening program. We report herein the results achieved to this end.

\section{Results and Discussion}

The contradictory data reported in last two decades [25-36], alerted us to be careful in our preparation of hydrazide 2, for instance the hours of reflux used in the title reaction were $2 \mathrm{~h}[28,33]$, $3 \mathrm{~h}$ [29], $4 \mathrm{~h} \mathrm{[30],} 6 \mathrm{~h} \mathrm{[27,36]}$ and $10 \mathrm{~h}$ [26,34], and afforded different yields of the claimed hydrazide 2: 99\% [25], 90\% [26], 80\% [27-30], 72\% [32], 65\% [31] and 62\% [34]. Moreover, different melting points have been reported for the claimed hydrazide 2 (m.p. 136-138 ${ }^{\circ} \mathrm{C}$ [26], $145{ }^{\circ} \mathrm{C}$ [32], 206-209 ${ }^{\circ} \mathrm{C}[30]$ ).

When we reacted an equimolar ratio of ester 1 with hydrazine hydrate at reflux and followed the reaction progress by TLC, the spot of ester 1 still remained, even after refluxing for $20 \mathrm{~h}$. However, the ester 1 was completely consumed after refluxing $2 \mathrm{~h}$ when we used excess hydrazine hydrate ( 2.5 equiv.). In this case, after cooling, we isolated yellow crystals with melting point $205-206^{\circ} \mathrm{C}$.

The IR, mass and NMR spectral data did not coincide however with those of the expected hydrazide 2. For example, the ${ }^{1} \mathrm{H}-\mathrm{NMR}$ of our yellow crystals revealed one $\mathrm{D}_{2} \mathrm{O}$-exchangeable signal, whereas there were no carbonyl resonances in the ${ }^{13} \mathrm{C}-\mathrm{NMR}$ data. In addition, its IR spectrum showed no evidence of the characteristic lactone carbonyl absorption band, while the mass spectrum of these crystals showed a peak at $\mathrm{m} / \mathrm{z} 240.5$ and not the expected one at $\mathrm{m} / \mathrm{z} 204$ corresponding to the molecular ion of claimed hydrazide 2 .

In an exhaustive literature survey of the reaction product(s) of ester $\mathbf{1}$ and hydrazine hydrate, bearing in consideration the possible ring-openings of chromene-2-ones [37], Soliman et al. [38] described, in 1985, the reaction of ethyl 2-oxo-2H-chromene-3-carboxylate (1) with hydrazine hydrate in refluxing ethanol, in which they obtained salicylaldehyde azine (3) in $31.5 \%$ and $42.4 \%$ yields for one and two equivalent of hydrazine hydrate, respectively, instead of the claimed hydrazide 2 . The structure of the well-known and commercially available salicylaldehyde azine (3), was rigorously proven by independent synthesis using the reaction of salicylaldehyde with hydrazine hydrate [39]. Comparison of the melting point and spectral data for an authentic sample of compound $\mathbf{3}$ and the yellow crystals produced in our trials, gave us absolute confidence to assign the structure of these crystals to be salicylaldehyde azine (3), in complete agreement with the results of Soliman and his coworkers (Scheme 1). 
Scheme 1. The reaction of ester 1 with hydrazine hydrate.

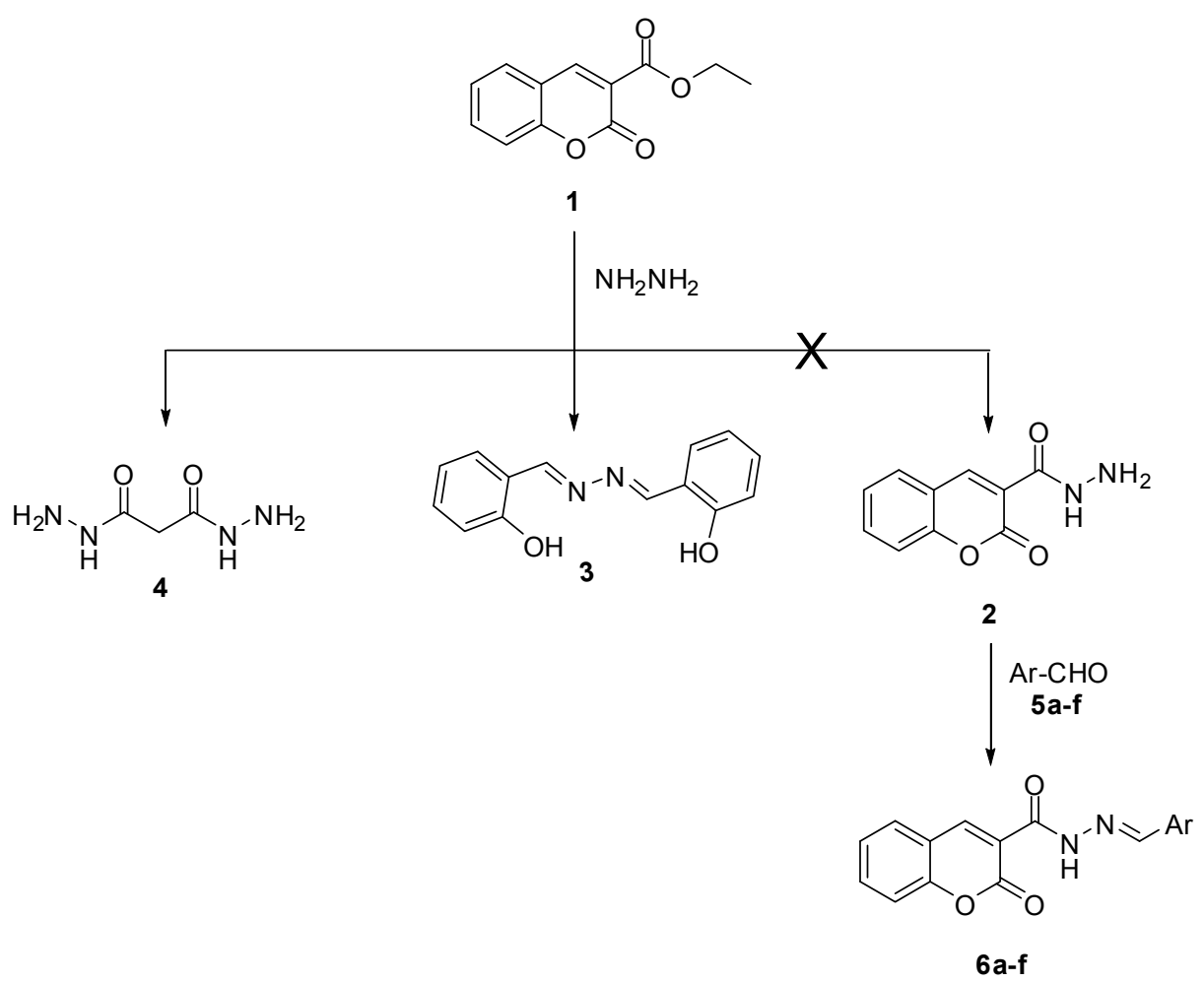

Although the azine 3, produced by Soliman et al. through the reaction of ester $\mathbf{1}$ with hydrazine hydrate, proved to be identical with the substance we have produced by the same reaction, in the current work, when the filtrate was evaporated under vacuum, after separation of salicylaldehyde azine (3), a white powder was produced, which was recrystallized from ethanol to give colorless flakes with mp. $153-154{ }^{\circ} \mathrm{C}$. ${ }^{1} \mathrm{H}-\mathrm{NMR}$ of the latter showed a singlet at $\delta 3.41$ integrating for $2 \mathrm{Hs}$, in addition to two $\mathrm{D}_{2} \mathrm{O}$-exchangeable signals at $\delta 4.38$ and $\delta 9.07$ integrating for $2 \mathrm{H}$ and $1 \mathrm{H}$, respectively, whereas its ${ }^{13} \mathrm{C}$-NMR spectrum showed only two peaks, the first of a sp ${ }^{3}$ carbon at $\delta 46.5$ and the second for two $\mathrm{sp}^{2}$ carbons at $\delta$ 170.7. The ${ }^{1} \mathrm{H}$ - or ${ }^{13} \mathrm{C}-\mathrm{NMR}$ spectra did not show any aromatic protons or carbons, respectively. The mass spectrum of the latter compound exhibited a peak at $m / z 132.3$.

Furthermore, on the basis of formation of salicylaldehyde azine (3) and according to the reported ring-opening behavior of the $2 \mathrm{H}$-chromene moiety, we can propose a mechanism for the formation of compound $\mathbf{3}$ and we can predict the structure of the obtained unknown compound in the title reaction. $2 \mathrm{H}$-1-Benzopyran-2-one (coumarin) derivatives are highly reactive because the pyran-2-one ring is an aliphatic moiety that is likely to undergo ring-opening under nucleophilic attack at the lactone acyl centre or nucleophilic conjugate addition at the carbon-carbon double bond [37]. Therefore, in ester 1 we have two nucleophilic centers in addition to the carbonyl function in the ester side chain (Scheme 2).

The previous facts led our proposed mechanism to suppose malonohydrazide (4) to be the structure of the compound isolated from the filtrate of the title reaction. In addition, comparing analytical data of the isolated malonohydrazide (4) with that of authentic sample of malonohydrazide (m.p. 151-153 ${ }^{\circ} \mathrm{C}$ ) [40] revealed them to be identical in all aspects. Moreover, the proposed mechanism proved the needed molar ratio of hydrazine hydrate $(2.5 \mathrm{~mol}$ equiv.) to achieve full consumption of ester $\mathbf{1}$. However, compounds 3 and 4 were also isolated when the reaction proceeded at $0-5{ }^{\circ} \mathrm{C}$. 
Scheme 2. The proposed mechanism for the reaction of ester 1 with hydrazine hydrate and its molar ratio equation.

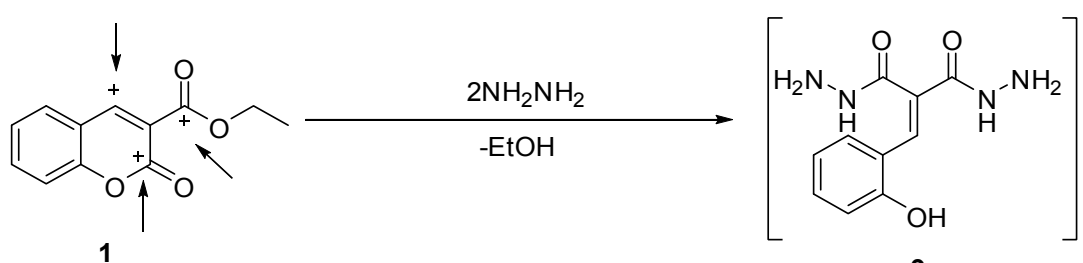

[9]| $\mathrm{NH}_{2} \mathrm{NH}_{2}$
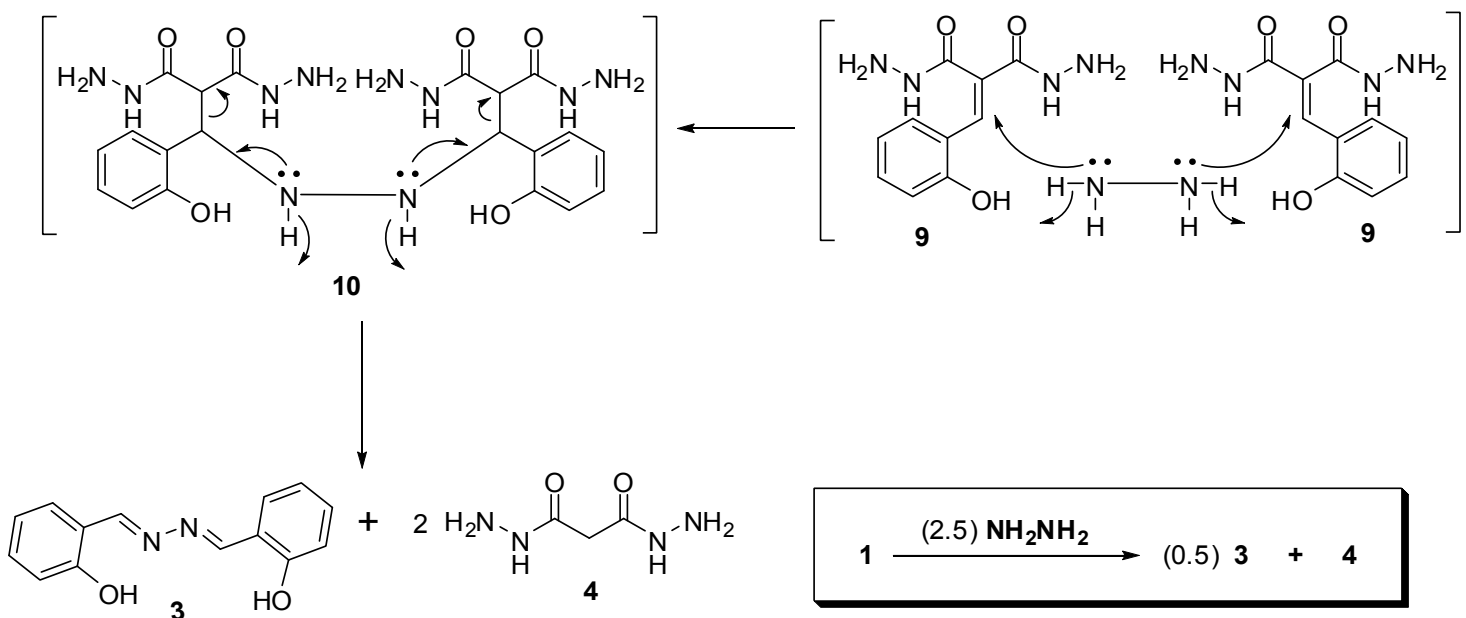

Our attention was next shifted to the synthesis of our targeted hydrazones $\mathbf{6 a}-\mathbf{f}$ (Scheme 1). Scheme 3 shows a representative retrosynthetic approach for this class of compounds. The ethyl 3-(2-arylidenehydrazinyl)-3-oxopropanoates $\mathbf{8 a - f}$ were condensed with salicylaldehyde (5f) to generate the targeted hydrazones $\mathbf{6 a}-\mathbf{f}$. Compounds $\mathbf{8 a}-\mathbf{f}$ were synthesized from the reaction of ethyl 3-hydrazinyl-3-oxopropanoate (7) with the appropriate aldehydes 5a-e.

Scheme 3. Retrosynthetic analysis of the targeted derivatives $\mathbf{6 a - f}$.

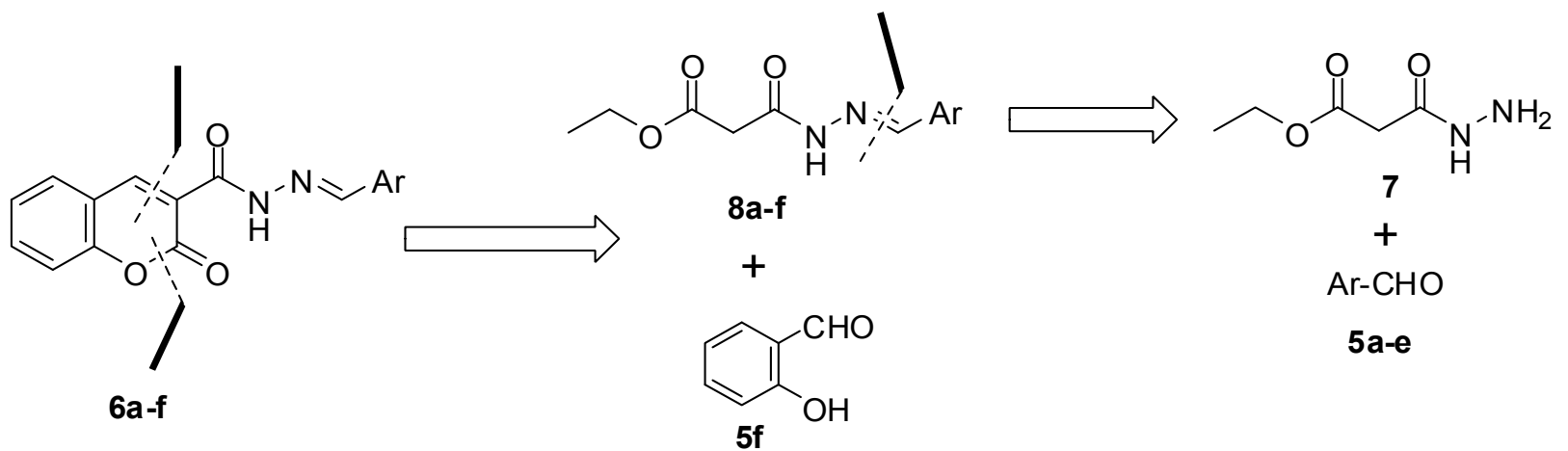

Thus, treatment of ethyl 3-hydrazinyl-3-oxopropanoate (7) [38] with the appropriate aldehydes 5a-f in refluxing ethanol yielded the corresponding ethyl 3-(2-arylidenehydrazinyl)-3-oxopropanoates 8a-f (Scheme 4). 
Scheme 4. Synthetic route for synthesis of hydrazones $\mathbf{6 a - f}$.

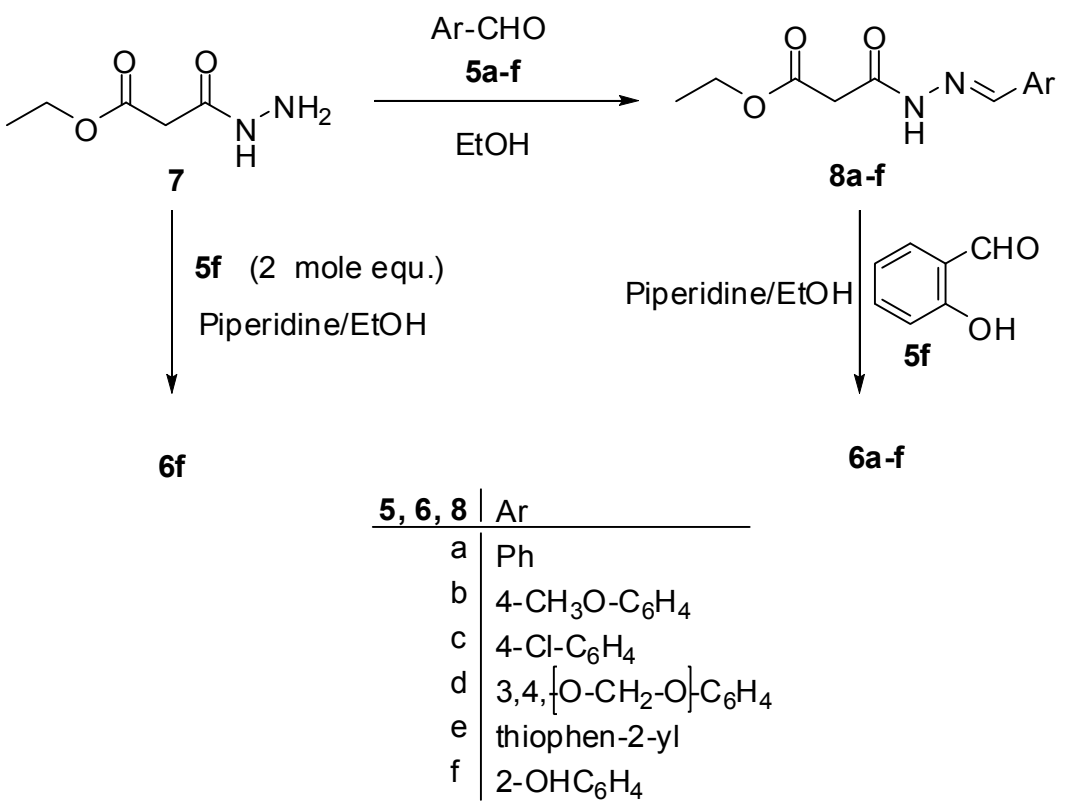

Next, the condensation reaction of hydrazones $\mathbf{8 a}-\mathbf{f}$ with salicyaldehyde $(\mathbf{5 f})$ in the presence of piperidine afforded exclusively compounds 6a-f. IR spectra of the latter products revealed two carbonyl absorption bands in the 1704-1699 and 1666-1610 $\mathrm{cm}^{-1}$ regions, in addition to the absorption band of NH function in the $3291-3195 \mathrm{~cm}^{-1}$ region. Their ${ }^{1} \mathrm{H}-\mathrm{NMR}$ spectra exhibited NH group $\mathrm{D}_{2} \mathrm{O}$-exchangeable signals in the $\delta 11.65-11.95$ region, in addition to two singlet signals in the $\delta$ $8.38-8.71$ and $\delta 8.90-8.94$ regions corresponding to the $-\mathrm{CH}=\mathrm{C}-$ group of the hydrazone function and pyran ring, respectively. In our hands, the melting points of $8 \mathbf{a}, 8 \mathbf{b}$ and $8 \mathbf{c}$ were recorded as $258-260{ }^{\circ} \mathrm{C}$, 265-267 ${ }^{\circ} \mathrm{C}$ and $288-290{ }^{\circ} \mathrm{C}$, respectively, while the reported melting points of the latter claimed compounds $205-207{ }^{\circ} \mathrm{C}, 194-196{ }^{\circ} \mathrm{C}$ and $216-218{ }^{\circ} \mathrm{C}$, respectively [30]. Finally, compound $\mathbf{6 f}$ was synthesized directly by the reaction of ethyl 3-hydrazinyl-3-oxopropanoate (7) with two mol equiv. of salicylaldehyde $\mathbf{( 5 f )}$ in the presence of piperidine.

\section{Experimental}

\subsection{General}

Infrared (IR) Spectra were recorded as $\mathrm{KBr}$ disks using the Perkin Elmer FT-IR Spectrum BX apparatus. Melting points were determined on a Gallenkamp melting point apparatus and are uncorrected. NMR Spectra were scanned in DMSO- $d_{6}$ on a Jeol NMR spectrophotometer operating at $400 \mathrm{MHz}$ for ${ }^{1} \mathrm{H}$ and $100 \mathrm{MHz}$ for ${ }^{13} \mathrm{C}$. Chemical shifts are expressed in $\delta$-values (ppm) relative to TMS as an internal standard. Coupling constants $(J)$ are expressed in $\mathrm{Hz} . \mathrm{D}_{2} \mathrm{O}$ was added to confirm the exchangeable protons. Mass spectra were measured on an Agilent Triple Quadrupole 6410 QQQ LC/MS equipped with an ESI (electrospray ionization) source. The elemental analyses were performed at the Microanalytical Center of Cairo University. Ethyl 3-hydrazinyl-3-oxopropanoate (7) was prepared according to the reported method (m.p. $68-69^{\circ} \mathrm{C}$ ) [40]. 


\subsection{The Reaction of Ethyl 2-oxo-2H-Chromene-3-carboxylate (1) with Hydrazine Hydrate}

A mixture of ethyl 2-oxo-2H-chromene-3-carboxylate $(1,2.18 \mathrm{~g}, 10 \mathrm{mmol})$ and hydrazine hydrate, $(99 \%, 1.3 \mathrm{~g}, 25 \mathrm{mmol})$ in absolute ethanol $(50 \mathrm{~mL})$ was heated under reflux for $2 \mathrm{~h}$. The precipitate formed was filtered off, washed with ethanol and dried. Recrystallization from ethanol gave yellow crystals of salicyaldehyde azine (3) [38,39] (1.15 g, 48\%); m.p. $205-206{ }^{\circ} \mathrm{C}\left(204.3{ }^{\circ} \mathrm{C}\right)$ [39]; IR $v$ 3350-2830 (OH), $1633(\mathrm{C}=\mathrm{N}) \mathrm{cm}^{-1} ;{ }^{1} \mathrm{H}-\mathrm{NMR} \delta$ 6.84-7.72 (m, 8H, ArH), $8.86(\mathrm{~s}, 2 \mathrm{H},-\mathrm{CH}=), 10.90$ (s, $\mathrm{D}_{2} \mathrm{O}$ exch., $\left.2 \mathrm{H}, \mathrm{OH}\right) ;{ }^{13} \mathrm{C}-\mathrm{NMR} \delta 117.9,118.7,121.4,132.0,132.5,159.2,162.0 ; \mathrm{MS} \mathrm{m} / z: 240.5$ $\left(\mathrm{M}^{+}\right)$. Anal. calcd. for $\mathrm{C}_{14} \mathrm{H}_{12} \mathrm{~N}_{2} \mathrm{O}_{2}$ (240.26): C, 69.99; H, 5.03; N, 11.66\%. Found: C, 70.23; H, 5.17; $\mathrm{N}, 11.48 \%$. Evaporation of the filtrate of the latter reaction gave a residue, which was washed with $70 \%$ ethanol, dried and recrystallized from ethanol to yield colorless flakes of malonohydrazide (4) [40] (1.14 g, 86\%); m.p. $153-154{ }^{\circ} \mathrm{C}\left(151-153{ }^{\circ} \mathrm{C}\right)$; IR v 3323-3246 $\left(\mathrm{NH}_{2}, \mathrm{NH}\right), 1635(\mathrm{C}=\mathrm{O}), \mathrm{cm}^{-1}$;

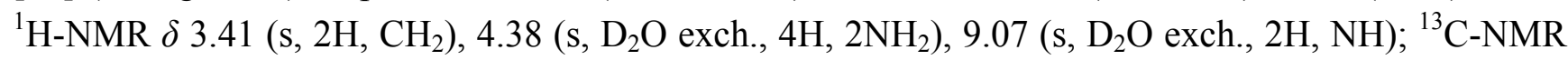
$\delta 46.5\left(\underline{\mathrm{CH}}_{2}\right), 170.7(2 \underline{\mathrm{C}}=\mathrm{O}) ; \mathrm{MS} m / z: 132.3\left(\mathrm{M}^{+}\right)$. Anal. calcd. for $\mathrm{C}_{3} \mathrm{H}_{8} \mathrm{~N}_{4} \mathrm{O}_{2}$ (132.12): $\mathrm{C}, 27.27 ; \mathrm{H}$, $6.10 ; \mathrm{N}, 42.41 \%$. Found: C, 27.04; H, 6.38; N, 42.16\%.

\subsection{General Procedure for the Synthesis of Ethyl 3-(2-arylidenehydrazinyl)-3-oxopropanoates 8a-f}

A solution of hydrazide $7(1.46 \mathrm{~g}, 10 \mathrm{mmol})$ and the appropriate aldehyde $5 \mathbf{a}-\mathbf{f}(10 \mathrm{mmol})$ in absolute ethanol $(30 \mathrm{~mL})$ was refluxed for $1 \mathrm{~h}$ and then cooled to $5-10{ }^{\circ} \mathrm{C}$. The solid formed was collected by filtration, washed with ethanol and crystallized from ethanol to afford hydrazones $\mathbf{8 a}-\mathbf{f}$.

Ethyl 3-(2-benzylidenehydrazinyl)-3-oxopropanoate (8a).Yield (2.0 g, 85\%); m.p. 115-17 ${ }^{\circ} \mathrm{C}$ (m.p. 111-113 ${ }^{\circ} \mathrm{C}$ [41]); IR v $3292(\mathrm{NH}), 1735(\mathrm{C}=\mathrm{O}$, ester $), 1682(\mathrm{C}=\mathrm{O}$, amide $) \mathrm{cm}^{-1}$; ${ }^{1} \mathrm{H}-\mathrm{NMR} \delta 1.17(\mathrm{t}$, $\left.J=6.6 \mathrm{~Hz}, 3 \mathrm{H}, \mathrm{CH}_{3}\right), 3.65\left(\mathrm{~s}, 2 \mathrm{H}, \mathrm{CH}_{2}\right), 4.09\left(\mathrm{q}, J=6.6 \mathrm{~Hz}, 2 \mathrm{H}, \mathrm{CH}_{2}\right), 7.41-7.71(\mathrm{~m}, 5 \mathrm{H}, \mathrm{ArH}), 7.96(\mathrm{~s}$, $1 \mathrm{H},-\mathrm{CH}=), 11.58$ (s, $\mathrm{D}_{2} \mathrm{O}$ exch., $\left.1 \mathrm{H}, \mathrm{NH}\right) ;{ }^{13} \mathrm{C}-\mathrm{NMR} \delta 14.6\left(\underline{\left.\mathrm{CH}_{3}\right),} 41.8\left(\underline{\mathrm{CH}}_{2}\right), 61.0\left(\underline{\mathrm{CH}}_{2}\right), 127,3\right.$, 129.2, 129,3, 129.8, $134.6(\mathrm{ArC}), 143.7(-\underline{\mathrm{C}} \mathrm{H}=), 168.3(\underline{\mathrm{C}}=\mathrm{O}), 168.5(\underline{\mathrm{C}}=\mathrm{O})$; MS m/z: $257.1\left(\mathrm{M}^{+}+23\right)$. Anal. calcd. for $\mathrm{C}_{12} \mathrm{H}_{14} \mathrm{~N}_{2} \mathrm{O}_{3}$ (234.25): C, 61.53; H, 6.02; N, 11.96\%. Found: C, 61.27; H, 5.85; N, $12.19 \%$.

Ethyl 3-(2-(4-methoxybenzylidene)hydrazinyl)-3-oxopropanoate (8b). Yield (2.46 g, 93\%); m.p. 116-118 ${ }^{\circ} \mathrm{C}$ (m.p. $110-111^{\circ} \mathrm{C}$ [41]); IR $v 3293(\mathrm{NH}), 1728\left(\mathrm{C}=\mathrm{O}\right.$, ester), $1681\left(\mathrm{C}=\mathrm{O}\right.$, amide) cm ${ }^{-1}$; ${ }^{1} \mathrm{H}-\mathrm{NMR} \delta 1.16\left(\mathrm{t}, J=6.6 \mathrm{~Hz}, 3 \mathrm{H}, \mathrm{CH}_{3}\right), 3.62\left(\mathrm{~s}, 2 \mathrm{H}, \mathrm{CH}_{2}\right), 3.80\left(\mathrm{~s}, 3 \mathrm{H}, \mathrm{OCH}_{3}\right), 4.08\left(\mathrm{~s}, 2 \mathrm{H}, \mathrm{CH}_{2}\right)$, 4.07 (q, $\left.J=6.6, \mathrm{~Hz}, 2 \mathrm{H}, \mathrm{CH}_{2}\right), 6.98$ (d, $\left.J=8.8, \mathrm{~Hz}, 2 \mathrm{H}, \mathrm{ArH}\right), 7.57$ (d, $\left.J=8.8 \mathrm{~Hz}, 2 \mathrm{H}, \mathrm{ArH}\right), 7.90$ (s, $1 \mathrm{H},-\mathrm{CH}=), 11.44$ (s, $\mathrm{D}_{2} \mathrm{O}$ exch., $\left.1 \mathrm{H}, \mathrm{NH}\right) ;{ }^{13} \mathrm{C}-\mathrm{NMR} \delta 14.6\left(\underline{\mathrm{CH}}_{3}\right), 41.7\left(\underline{\left.\mathrm{CH}_{2}\right),} 55.9\left(\mathrm{OCH}_{3}\right), 60.9\right.$ $\left(\underline{\mathrm{CH}}_{2}\right), 114.8,127.2,128.9,(\mathrm{ArC}), 143.5(-\underline{\mathrm{CH}}=), 161.2(\mathrm{ArC}), 168.2(\underline{\mathrm{C}}=\mathrm{O}), 168.3(\underline{\mathrm{C}}=\mathrm{O}) ; \mathrm{MS} \mathrm{m} / z$ : $265.2\left(\mathrm{M}^{+}+1\right), 287.2\left(\mathrm{M}^{+}+23\right)$. Anal. calcd. for $\mathrm{C}_{13} \mathrm{H}_{16} \mathrm{~N}_{2} \mathrm{O}_{4}$ (264.28): C, 59.08; H, 6.10; N, 10.60\%. Found: C, 60.24; H, 6.36; N, 10.57\%.

Ethyl 3-(2-(4-chlorobenzylidene)hydrazinyl)-3-oxopropanoate $(\mathbf{8 c})$. Yield (2.23 g, 83\%); m.p. 168-170 ${ }^{\circ} \mathrm{C}$ (m.p. $160-163{ }^{\circ} \mathrm{C}$ [41]); IR $v 3292(\mathrm{NH}), 1733\left(\mathrm{C}=\mathrm{O}\right.$, ester), $1675\left(\mathrm{C}=\mathrm{O}\right.$, amide) $\mathrm{cm}^{-1}$; ${ }^{1} \mathrm{H}-\mathrm{NMR} \delta$ $1.16\left(\mathrm{t}, J=6.6 \mathrm{~Hz}, 3 \mathrm{H}, \mathrm{CH}_{3}\right), 3.65\left(\mathrm{~s}, 2 \mathrm{H}, \mathrm{CH}_{2}\right), 4.08\left(\mathrm{q}, J=6.6 \mathrm{~Hz}, 2 \mathrm{H}, \mathrm{CH}_{2}\right), 7.49$ (d, $J=8.8 \mathrm{~Hz}, 2 \mathrm{H}, \mathrm{ArH}), 7.65(\mathrm{~d}, J=8.1, \mathrm{~Hz}, 2 \mathrm{H}, \mathrm{ArH}), 7.96(\mathrm{~s}, 1 \mathrm{H},-\mathrm{CH}=), 11.64\left(\mathrm{~s}, \mathrm{D}_{2} \mathrm{O}\right.$ exch., $1 \mathrm{H}$, 
$\mathrm{NH}) ;{ }^{13} \mathrm{C}-\mathrm{NMR} \delta 14.6\left(\underline{\mathrm{CH}}_{3}\right), 41.5\left(\underline{\mathrm{CH}}_{2}\right), 61.1\left(\underline{\mathrm{CH}}_{2}\right), 128.9,129.4,133.6,134.9$ (ArC), 142.4 $(-\underline{\mathrm{CH}}=), 168.2(\underline{\mathrm{C}}=\mathrm{O}), 168.5(\underline{\mathrm{C}}=\mathrm{O}) ; \mathrm{MS} m / z: 291.1\left(\mathrm{M}^{+}+23\right)$. Anal. calcd. for $\mathrm{C}_{12} \mathrm{H}_{13} \mathrm{ClN}_{2} \mathrm{O}_{3}(268.70)$ : C, 53.64; H, 4.88; N, 10.43\%. Found: C, 53.75; H, 5.04; N, 10.25\%.

Ethyl 3-(2-(benzo[d][1,3]dioxol-5-ylmethylene)hydrazinyl)-3-oxopropanoate (8d). Yield (2.37 g, 85\%); m.p. 158-160 ${ }^{\circ} \mathrm{C}$; IR $v 3187(\mathrm{NH}), 1736(\mathrm{C}=\mathrm{O}$, ester $), 1676\left(\mathrm{C}=\mathrm{O}\right.$, amide) $\mathrm{cm}^{-1}$; ${ }^{1} \mathrm{H}-\mathrm{NMR} \delta 1.17$ (t, $\left.J=7.36 \mathrm{~Hz}, 3 \mathrm{H}, \mathrm{CH}_{3}\right), 3.61\left(\mathrm{~s}, 2 \mathrm{H}, \mathrm{CH}_{2}\right), 4.07\left(\mathrm{q}, J=7.36 \mathrm{~Hz}, 2 \mathrm{H}, \mathrm{CH}_{2}\right), 6.07\left(\mathrm{~s}, 2 \mathrm{H}, \mathrm{CH}_{2}\right), 6.95(\mathrm{~d}$, $J=8.1 \mathrm{~Hz}, 1 \mathrm{H}, \mathrm{ArH}), 7.06(\mathrm{~d}, J=8.8 \mathrm{~Hz}, 1 \mathrm{H}, \mathrm{ArH}), 7.22(\mathrm{~s}, 1 \mathrm{H}, \mathrm{ArH}), 7.86(\mathrm{~s}, 1 \mathrm{H},-\mathrm{CH}=), 11.46$ (s, $\mathrm{D}_{2} \mathrm{O}$ exch., $\left.1 \mathrm{H}, \mathrm{NH}\right) ;{ }^{13} \mathrm{C}-\mathrm{NMR} \delta 14.6\left(\underline{\mathrm{CH}}_{3}\right), 41.8\left(\underline{\mathrm{CH}}_{2}\right), 60.9\left(\underline{\mathrm{CH}}_{2}\right), 102.1\left(\underline{\mathrm{CH}}_{2}\right), 105.2,108.9$, 123.7, 129.1 (ArC), 143.3 ( $-\underline{\mathrm{C}} \mathrm{H}=), 148.5,149.5$ (ArC), $168.30(\underline{\mathrm{C}}=\mathrm{O}), 168.33(\underline{\mathrm{C}}=\mathrm{O}) ; \mathrm{MS} m / z: 279.1$ $\left(\mathrm{M}^{+}+1\right)$, $301.1\left(\mathrm{M}^{+}+23\right)$. Anal. calcd. for $\mathrm{C}_{13} \mathrm{H}_{14} \mathrm{~N}_{2} \mathrm{O}_{5}$ (278.26): C, 56.11; H, 5.07; N, 10.07\%. Found: C, 56.19; H, 4.93; N, 9.86\%.

Ethyl 3-oxo-3-(2-(thiophen-2-ylmethylene)hydrazinyl)propanoate (8e). Yield (1.87 g, 78\%); m.p. 118-120 ${ }^{\circ} \mathrm{C}$; IR $v 3292(\mathrm{NH}), 1732\left(\mathrm{C}=\mathrm{O}\right.$, ester), $1677\left(\mathrm{C}=\mathrm{O}\right.$, amide) $\mathrm{cm}^{-1} ;{ }^{1} \mathrm{H}-\mathrm{NMR} \delta 1.19$ (t, $J=7.32 \mathrm{~Hz}$, $\left.3 \mathrm{H}, \mathrm{CH}_{3}\right), 3.57\left(\mathrm{~s}, 2 \mathrm{H}, \mathrm{CH}_{2}\right), 4.09$ (q, $\left.J=7.32 \mathrm{~Hz}, 2 \mathrm{H}, \mathrm{CH}_{2}\right), 8.14(\mathrm{~s}, 1 \mathrm{H},-\mathrm{CH}=), 7.10$ (t, $J=4.4 \mathrm{~Hz}$, $1 \mathrm{H}$, thiophene $\mathrm{H}), 7.40(\mathrm{~d}, J=2.92 \mathrm{~Hz}, 1 \mathrm{H}$, thiophene $\mathrm{H}), 7.62(\mathrm{~d}, J=4.4 \mathrm{~Hz}, 1 \mathrm{H}$, thiophene $\mathrm{H}) ; 11.56$ (s, $\mathrm{D}_{2} \mathrm{O}$ exch., $\left.1 \mathrm{H}, \mathrm{NH}\right)$; $\mathrm{MS} m / z$ : $241.1\left(\mathrm{M}^{+}+1\right), 263.1\left(\mathrm{M}^{+}+23\right)$. Anal. calcd. for $\mathrm{C}_{10} \mathrm{H}_{12} \mathrm{~N}_{2} \mathrm{O}_{3} \mathrm{~S}$ (240.28): C, 49.99; H, 5.03; N, 11.66; S, 13.34\%. Found: C, 49.79; H, 5.07; N, 11.86; S, 13.53\%.

Ethyl 3-(2-(2-hydroxybenzylidene)hydrazinyl)-3-oxopropanoate (8f). Yield (2.15 g, 86\%); m.p. 148-150 ${ }^{\circ} \mathrm{C}$; IR v 3191-3090 (NH, OH), $1734\left(\mathrm{C}=\mathrm{O}\right.$, ester), $1667\left(\mathrm{C}=\mathrm{O}\right.$, amide) $\mathrm{cm}^{-1}$; ${ }^{1} \mathrm{H}-\mathrm{NMR} \delta 1.17$ (t, $\left.J=7.32 \mathrm{~Hz}, 3 \mathrm{H}, \mathrm{CH}_{3}\right), 3.62\left(\mathrm{~s}, 2 \mathrm{H}, \mathrm{CH}_{2}\right), 4.09$ (q, $\left.J=7.32 \mathrm{~Hz}, 2 \mathrm{H}, \mathrm{CH}_{2}\right), 6.82-6.93(\mathrm{~m}, 2 \mathrm{H}, \mathrm{ArH})$, 7.21-7.31 (m, 1H, ArH), 7.54-7.62 (m, 1H, ArH), 8.27 (s, 1H, -CH=), 9.99 (s, $\mathrm{D}_{2} \mathrm{O}$ exch., 1H, OH), 11.49 (s, $\mathrm{D}_{2} \mathrm{O}$ exch., $\left.1 \mathrm{H}, \mathrm{NH}\right) ;{ }^{13} \mathrm{C}-\mathrm{NMR} \delta 14.6\left(\underline{\mathrm{CH}}_{3}\right), 41.7\left(\underline{\mathrm{CH}}_{2}\right), 61.0\left(\underline{\mathrm{CH}}_{2}\right), 116.7,119.9,120.7$, 126.5, 131.7 (ArC), $141.1(-\underline{\mathrm{C}}=), 162.1,(\mathrm{ArC}), 168.1(\underline{\mathrm{C}}=\mathrm{O}), 168.2(\underline{\mathrm{C}}=\mathrm{O}) ; \mathrm{MS} m / z: 251.2\left(\mathrm{M}^{+}+1\right)$, $273.1\left(\mathrm{M}^{+}+23\right)$. Anal. calcd. for $\mathrm{C}_{12} \mathrm{H}_{14} \mathrm{~N}_{2} \mathrm{O}_{4}$ (250.25): C, 57.59; H, 5.64; N, 11.19\%. Found: C, 57.46; $\mathrm{H}, 5.67 ; \mathrm{N}, 11.03 \%$.

\subsection{General Procedure for the Synthesis of N'-Arylidene-2-oxo-2H-chromene-3-carbohydrazides 6a-f}

To a solution of the appropriate hydrazone $\mathbf{8 a}-\mathbf{f}(1 \mathrm{mmol})$ and salicaldehyde $(\mathbf{5 f})(0.122 \mathrm{~g}, 1 \mathrm{mmol})$ in absolute ethanol $(25 \mathrm{~mL})$, a catalytic amount of piperidine $(0.3 \mathrm{~mL})$ was added. The reaction mixture was refluxed for $1 \mathrm{~h}$. The formed precipitate was filtered off, washed with ethanol, dried and recrystallized from EtOH/DMF to give hydrazides $\mathbf{6 a}-\mathbf{f}$.

$N^{\prime}$-Benzylidene-2-oxo-2H-chromene-3-carbohydrazide (6a). Yield (254 mg, 87\%); m.p. 258-260 ${ }^{\circ} \mathrm{C}$; IR $v 3249(\mathrm{NH}), 1700\left(\mathrm{C}=\mathrm{O}\right.$, lactone), $1665\left(\mathrm{C}=\mathrm{O}\right.$, amide) $\mathrm{cm}^{-1} ;{ }^{1} \mathrm{H}-\mathrm{NMR} \delta$ 7.46-7.51 (m, 4H, ArH), $7.55(\mathrm{~d}, J=8.1 \mathrm{~Hz}, 1 \mathrm{H}, \operatorname{ArH}), 7.76-7.77$ (m, 3H, ArH), 8.03 (d, $J=8.1 \mathrm{~Hz}, 1 \mathrm{H}, \operatorname{ArH}), 8.48$ (s, 1H, $-\mathrm{CH}=), 8.93(\mathrm{~s}, 1 \mathrm{H},-\mathrm{CH}=), 11.78\left(\mathrm{~s}, \mathrm{D}_{2} \mathrm{O}\right.$ exch., $\left.1 \mathrm{H}, \mathrm{NH}\right) ;{ }^{13} \mathrm{C}-\mathrm{NMR} \delta 116.8,116.9,118.9,119.9$, 125.8, 127.2, 127.9, 129.4, 130.9, 131.0, 134.5, 134.9, 145.2 (ArC), 148.2 ( $-\underline{\mathrm{C}} \mathrm{H}=), 149.9$ ( $-\underline{\mathrm{C}} \mathrm{H}=), 154.5$ $(\underline{\mathrm{C}}=\mathrm{O})$; MS $m / z$ : $293.1\left(\mathrm{M}^{+}+1\right), 315.1\left(\mathrm{M}^{+}+23\right)$. Anal. calcd. for $\mathrm{C}_{17} \mathrm{H}_{12} \mathrm{~N}_{2} \mathrm{O}_{3}$ (292.29): $\mathrm{C}, 69.86 ; \mathrm{H}$, 4.14; N, 9.58\%. Found: C, 69.58; H, 4.03; N, 9.36\%. 
$N^{\prime}$-(4-Methoxybenzylidene)-2-oxo-2H-chromene-3-carbohydrazide (6b). Yield (290 mg, 90\%); m.p. 265-267 ${ }^{\circ} \mathrm{C}$; IR $v 3257(\mathrm{NH}), 1704\left(\mathrm{C}=\mathrm{O}\right.$, lactone), $1666\left(\mathrm{C}=\mathrm{O}\right.$, amide) $\mathrm{cm}^{-1}$; ${ }^{1} \mathrm{H}-\mathrm{NMR} \delta 3.82(\mathrm{~s}, 3 \mathrm{H}$, $\left.\mathrm{OCH}_{3}\right), 7.04(\mathrm{~d}, J=8.8 \mathrm{~Hz}, 2 \mathrm{H}, \mathrm{ArH}), 7.48$ (t, $\left.J=7.4 \mathrm{~Hz}, 1 \mathrm{H}, \mathrm{ArH}\right), 7.55$ (d, $\left.J=8.8 \mathrm{~Hz}, 1 \mathrm{H}, \mathrm{ArH}\right), 7.71$ (d, $J=8.1 \mathrm{~Hz}, 2 \mathrm{H}, \mathrm{ArH}), 7.79$ (t, $J=8.1 \mathrm{~Hz}, 1 \mathrm{H}, \mathrm{ArH}), 7.02$ (d, $J=7.3 \mathrm{~Hz}, 1 \mathrm{H}, \mathrm{ArH}), 8.40$ (s, 1H, $-\mathrm{CH}=), 8.90(\mathrm{~s}, 1 \mathrm{H},-\mathrm{CH}=), 11.65\left(\mathrm{~s}, \mathrm{D}_{2} \mathrm{O}\right.$ exch., $\left.1 \mathrm{H}, \mathrm{NH}\right)$; $\mathrm{MS} m / z: 323.2\left(\mathrm{M}^{+}+1\right)$. Anal. calcd. for $\mathrm{C}_{18} \mathrm{H}_{14} \mathrm{~N}_{2} \mathrm{O}_{4}$ (322.31): C, 67.07; H, 4.38; N, 8.69\%. Found: C, 66.79; H, 4.44; N, 8.75\%.

$N^{\prime}$-(4-Chlorobenzylidene)-2-oxo-2H-chromene-3-carbohydrazide (6c). Yield (265 mg, 81\%); m.p. 288-290 ${ }^{\circ} \mathrm{C}$; IR $v 3250(\mathrm{NH}), 1704\left(\mathrm{C}=\mathrm{O}\right.$, lactone), $1665\left(\mathrm{C}=\mathrm{O}\right.$, amide) $\mathrm{cm}^{-1} ; 6.95(\mathrm{~d}, J=7.4 \mathrm{~Hz}, 1 \mathrm{H}$, $\operatorname{ArH}), 7.48(\mathrm{t}, J=7.3 \mathrm{~Hz}, 2 \mathrm{H}, \mathrm{ArH}), 7.55$ (d, $J=8.1 \mathrm{~Hz}, 2 \mathrm{H}, \mathrm{ArH}), 7.78$ (d, $J=8.1 \mathrm{~Hz}, 2 \mathrm{H}, \operatorname{ArH}), 8.02$ (d, $J=6.6 \mathrm{~Hz}, 1 \mathrm{H}, \mathrm{ArH}), 8.49$ (s, 1H, $-\mathrm{CH}=), 8.94$ (s, 1H, $-\mathrm{CH}=), 11.95$ (s, $\mathrm{D}_{2} \mathrm{O}$ exch., 1H, NH); MS m/z: $327.1\left(\mathrm{M}^{+}+1\right)$. Anal. calcd. for $\mathrm{C}_{17} \mathrm{H}_{11} \mathrm{ClN}_{2} \mathrm{O}_{3}$ (326.73): C, 62.49; H, 3.39; N, 8.57\%. Found: C, $62.31 ; \mathrm{H}, 3.39 ; \mathrm{N}, 8.48 \%$.

$N^{\prime}$-(Benzo[d][1,3]dioxol-5-ylmethylene)-2-oxo-2H-chromene-3-carbohydrazide (6d). Yield (259 mg, 77\%); m.p. 286-288 ${ }^{\circ} \mathrm{C}$; IR $v 3256(\mathrm{NH}), 1704\left(\mathrm{C}=\mathrm{O}\right.$, lactone), $1665\left(\mathrm{C}=\mathrm{O}\right.$, amide $\mathrm{cm}^{-1}$; ${ }^{1} \mathrm{H}-\mathrm{NMR} \delta 6.11\left(\mathrm{~s}, 2 \mathrm{H}, \mathrm{CH}_{2}\right), 6.94-7.79(\mathrm{~m}, 6 \mathrm{H}, \mathrm{ArH}), 8.02(\mathrm{~d}, J=6.6 \mathrm{~Hz}, 1 \mathrm{H}, \mathrm{ArH}), 8.38(\mathrm{~s}, 1 \mathrm{H}$, $-\mathrm{CH}=), 8.92(\mathrm{~s}, 1 \mathrm{H},-\mathrm{CH}=), 11.69\left(\mathrm{~s}, \mathrm{D}_{2} \mathrm{O}\right.$ exch., $\left.1 \mathrm{H}, \mathrm{NH}\right)$; $\mathrm{MS} m / z: 337.1\left(\mathrm{M}^{+}+1\right)$. Anal. calcd. for $\mathrm{C}_{18} \mathrm{H}_{12} \mathrm{~N}_{2} \mathrm{O}_{5}$ (336.30): C, 64.29; H, 3.60; N, 8.33\%. Found: C, 64.02; H, 3.54; N, 8.52\%.

2-Oxo-N'-(thiophen-2-ylmethylene)-2H-chromene-3-carbohydrazide (6e). Yield (239 mg, 80\%); m.p. 282-284 ${ }^{\circ} \mathrm{C}$; IR $v 3291(\mathrm{NH}), 1700\left(\mathrm{C}=\mathrm{O}\right.$, lactone), $1664\left(\mathrm{C}=\mathrm{O}\right.$, amide $\mathrm{cm}^{-1} ;{ }^{1} \mathrm{H}-\mathrm{NMR} \delta 7.45-7.78$ (m, 6H, thiophene H, ArH), $8.02(\mathrm{~d}, J=8.1 \mathrm{~Hz}, 1 \mathrm{H}, \mathrm{ArH}), 8.69(\mathrm{~s}, 1 \mathrm{H},-\mathrm{CH}=), 8.91(\mathrm{~s}, 1 \mathrm{H},-\mathrm{CH}=)$, 11.85 (s, $\mathrm{D}_{2} \mathrm{O}$ exch., $\left.1 \mathrm{H}, \mathrm{NH}\right) ;{ }^{13} \mathrm{C}-\mathrm{NMR} \delta 116.8$ ( $\left.\underline{\mathrm{CH}}=\right), 118.9,119.9,125.8,128.6,130,1,130.8$, 132.3, 134.9, 139.1 (ArC, thiophene C), 145.1 ( $-\underline{\mathrm{C}} \mathrm{H}=), 148.3,154.4(\mathrm{ArC}), 158.5(\underline{\mathrm{C}}=\mathrm{O}), 160.4$ $(\underline{\mathrm{C}}=\mathrm{O})$; MS m/z: $299.1\left(\mathrm{M}^{+}+1\right)$. Anal. calcd. for $\mathrm{C}_{15} \mathrm{H}_{10} \mathrm{~N}_{2} \mathrm{O}_{3} \mathrm{~S}$ (298.32): C, 60.39; H, 3.38; N, 9.39; $\mathrm{S}$, $10.75 \%$. Found: C, 60.36; H, 3.15; N, 9.31; S, 10.59\%.

$N^{\prime}$-(2-Hydroxybenzylidene)-2-oxo-2H-chromene-3-carbohydrazide (6f). Yield (271 mg, 88\%); m.p. 298-300 ${ }^{\circ} \mathrm{C}$; IR $v$ 3195-3049 (NH, OH), 1699 (C=O, lactone), $1610\left(\mathrm{C}=\mathrm{O}\right.$, amide) $\mathrm{cm}^{-1}$; ${ }^{1} \mathrm{H}-\mathrm{NMR} \delta$ $6.96(\mathrm{~d}, J=6.6 \mathrm{~Hz}, 2 \mathrm{H}, \mathrm{ArH}), 7.33(\mathrm{t}, J=8.1 \mathrm{~Hz}, 1 \mathrm{H}, \mathrm{ArH}), 7.48$ (t, $J=8.1 \mathrm{~Hz}, 1 \mathrm{H}, \mathrm{ArH}), 7.55-7.57$ (m, 3H, ArH, OH), 7.79 (t, $J=8.1 \mathrm{~Hz}, 1 \mathrm{H}, \mathrm{ArH}), 8.02(\mathrm{~d}, J=7.3 \mathrm{~Hz}, 1 \mathrm{H}, \mathrm{ArH}), 0.00(\mathrm{~s}, 1 \mathrm{H},-\mathrm{CH}=)$, $8.71(\mathrm{~s}, 1 \mathrm{H},-\mathrm{CH}=), 8.92(\mathrm{~s}, 1 \mathrm{H},-\mathrm{CH}=)$; $\mathrm{MS} \mathrm{m} / z: 309.2\left(\mathrm{M}^{+}+1\right), 331.1\left(\mathrm{M}^{+}+23\right)$. Anal. calcd. for $\mathrm{C}_{17} \mathrm{H}_{12} \mathrm{~N}_{2} \mathrm{O}_{4}$ (308.29): C, 66.23; H, 3.92; N, 9.09\%. Found: C, 66.02; H, 4.11; N, 8.92\%.

\subsection{Direct Synthesis of $\mathbf{6} \mathbf{f}$}

To a solution of hydrazide $7(0.146 \mathrm{~g}, 1 \mathrm{mmol})$ and salicaldehyde $(\mathbf{5 f})(0.244 \mathrm{~g}, 2 \mathrm{mmol})$ in absolute ethanol $(25 \mathrm{~mL})$, a catalytic amount of piperidine $(0.3 \mathrm{~mL})$ was added. The reaction mixture was refluxed for $1 \mathrm{~h}$. The formed precipitate was filtered off, washed with ethanol, dried and recrystallized from $\mathrm{EtOH} / \mathrm{DMF}$ to give $240 \mathrm{mg}$ of $\mathbf{6} \mathbf{f}$ in $78 \%$ yield. 


\section{Conclusions}

We have identified another main product, confirmed as malohydrazide (4), besides salicylaldehyde azine (3) which was reported by Soliman et al. in 1985 [38] as the sole product of the title reaction. Whereas the observations of this investigation have confirmed the identity of salicylaldehyde azine (3) and malonohydrazide (4), as the compounds produced through the reaction of ester $\mathbf{1}$ with hydrazine hydrate, the structures of products claimed to be formed from hydrazide $\mathbf{2}$ in investigations reported over the last two decades [25-36] need to be reassigned. Moreover, we described an efficient synthetic route for our targeted hydrazones $\mathbf{6 a}-\mathbf{f}$. Generalization of the latter established method can be widely used in synthesis of library of coumarin-based hydrazones.

\section{Acknowledgments}

The authors extend their appreciation to the Deanship of Scientific Research at King Saud University for funding this work through research group No. RGP-VPP-196.

\section{References}

1. Fang, G.-M.; Li, Y.-M.; Shen, F.; Huang, Y.-C.; Li, J.-B.; Lin, Y.; Cui, H.-K.; Liu, L. Protein chemical synthesis by ligation of peptide hydrazides. Angew. Chem. Int. Ed. 2011, 50, 7645-7649.

2. Hassan, A.A.; Shawky, A.M. Chemistry and heterocyclization of carbohydrazides. J. Het. Chem. 2010, 47, 745-763.

3. Attanasi, O.A.; Filippone, P.; Perrulli, F.R.; Santeusanio, S. Regioselective role of the hydrazide moiety in the formation of complex pyrrole-pyrazole systems. Tetrahedron 2001, 57, 1387-1394.

4. Abdel-Aziz, M.; Abuo-Rahma, G.A.; Hassan, A.A. Synthesis of novel pyrazole derivatives and evaluation of their antidepressant and anticonvulsant activities. Eur. J. Med. Chem. 2009, 44, 3480-3487.

5. Mamolo, M.G.; Falagiani, V.; Zampieri, D.; Vio, L.; Banfi, E.; Scialino, G. Synthesis and antimycobacterial activity of (3,4-diaryl-3H-thiazol-2-ylidene)-hydrazide derivatives. IL Farmaco 2003, 58, 631-637.

6. Jha, K.K.; Samad, A.; Kumar, Y.; Shaharyar, M.; Khosa, R.L.; Jain, J.; Kumar, V.; Singh, P. Design, synthesis and biological evaluation of 1,3,4-oxadiazole derivatives. Eur. J. Med. Chem. 2010, 45, 4963-4967.

7. Yeung, K.-S.; Farkas, M.E.; Kadow, J.F.; Meanwell, N.A. A base-catalyzed, direct synthesis of 2,5-disubstituted 1,2,4-triazoles from nitriles and hydrazides. Tetrahedron Lett. 2005, 46, 3429-3432.

8. Farshori, N.N.; Banday, M.R.; Ahmad, A.; Khan, A.U.; Rauf, A. Synthesis, characterization, and in vitro antimicrobial activities of 5-alkenyl/hydroxyalkenyl-2-phenylamine-1,3,4-oxadiazoles and thiadiazoles. Bioorg. Med. Chem. Lett. 2010, 20, 1933-1938.

9. Almajan, G.L.; Barbuceanu, S.-F.; Bancescu, G.; Saramet, I.; Saramet, G.; Draghici, C. Synthesis and antimicrobial evaluation of some fused heterocyclic $[1,2,4]$ triazolo[3,4- $b][1,3,4]$ thiadiazole derivatives. Eur. J. Med Chem. 2010, 45, 6139-6146. 
10. Skoumbourdis, A.P.; Huang, R.; Southall, N.; Leister, W.; Guo, V.; Cho, M.-H.; Inglese, J.; Nirenberg, M.; Austin, C.P.; Xia, M.; et al. Identification of a potent new chemotype for the selective inhibition of PDE4. Bioorg. Med. Chem. Lett. 2008, 18, 1297-1303.

11. Saha, A.; Kumar, R.; Kumar, R.; Devakumar, C. Development and assessment of green synthesis of hydrazides. Indian J. Chem. 2010, 49B, 526-531.

12. Aboul-Fadl, T.; Abdel-Aziz, H.A.; Kadi, A.; Bari, A.; Ahmad, P.; Al-Samani, T.; Ng, S.W. Microwave-assisted one-step synthesis of fenamic acid hydrazides from the corresponding acids. Molecules 2011, 16, 3544-3551.

13. Chimenti, F.; Secci, D.; Bolasco, A.; Chimenti, P.; Bizzarri, B.; Granese, A.; Carradori, S.; Yáñez, M.; Orallo, F.; Ortuso, F.; et al. Synthesis, molecular modeling, and selective inhibitory activity against human monoamine oxidases of 3-carboxamido-7-substituted coumarins. J. Med. Chem. 2009, 52, 1935-1942.

14. Traven, V.F. New synthetic routes to furocoumarins and their analogs: A review. Molecules 2004, 9, 50-66.

15. Lacy, A.; O'Kennedy, R. Studies on coumarins and coumarin-related compounds to determine their therapeutic role in the treatment of cancer. Curr. Pharm. Des. 2004, 10, 3797-3811.

16. Fun, H.-K.; Attia, M.I.; Chia, T.S.; Elsaman, T.; Abdel-Aziz, H.A. 2-(2,3Dimethylanilino)benzohydrazide. Acta Cryst. 2012, E68, o2527-02528.

17. Abdel-Aziz, H.A.; Abdel-Wahab, B.F.; Badria, F.A. Stereoselective synthesis and antiviral activity of (1E,2Z,3E)-1-(piperidin-1-yl)-1-(arylhydrazono)-2-[(benzoyl/benzothiazol-2-oyl)hydrazono]4-(aryl $\left.{ }^{1}\right)$ but-3-enes. Arch. Pharm. 2010, 343, 152-159.

18. Abdel-Aziz, H.A.; Mekawey, A.A.I.; Dawood, K.M. Convenient synthesis and antimicrobial evaluation of some novel 2-substituted-3-methylbenzofuran derivatives. Eur. J. Med. Chem. 2009, 44, 3637-3644.

19. Abdel-Aziz, H.A.; Gamal-Eldeen, A.M.; Hamdy, N.A.; Fakhr, I.M.I. Immunomodulatory and anti-cancer activity of some novel 2-substituted-6-bromo-3-methylthiazolo[3,2-a]benzimidazole derivatives. Arch. Pharm. 2009, 342, 230-237.

20. Abdel-Wahab, B.F.; Abdel-Aziz, H.A.; Ahmed, E.M. Synthesis and antimicrobial evaluation of some new 1,3-thiazole, 1,3,4-thiadiazole, 1,2,4-triazole and [1,2,4]triazolo[3,4- $b][1,3,4]$ thiadiazine derivatives including 5-(benzofuran-2-yl)-1-phenyl-pyrazole moiety. Monatsh. Chem. 2009, 140, 601-605.

21. Abdel-Wahab, B.F.; Abdel-Aziz, H.A.; Ahmed, E.M. Convenient synthesis and antimicrobial activity of some new 3-substituted-5-(benzofuran-2-yl)-pyrazole derivatives. Arch. Pharm. 2008, 341, 734-739.

22. Abdel-Aziz, H.A.; Hamdy, N.A.; Farag, A.M.; Fakhr, I.M.I. Synthesis and reactions of 3-methylthiazolo[3,2-a]benzimidazole-2-carboxylic acid hydrazide: Synthesis of some new pyrazole, 1,3-thiazoline, 1,2,4-triazole and 1,2,4-triazolo[3,4-b]-1,3,4-thiadiazine derivatives pendant to thiazolo[3,2-a]benzimidazole moiety. J. Chin. Chem. Soc. 2007, 54, 1573-1582.

23. Dawood, K.M.; Farag, A.M.; Abdel-Aziz, H.A. Synthesis of some new benzofuran-based thiophene, 1,3-oxathiole and 1,3,4-oxa(thia)diazole derivatives. Heteroatom Chem. 2007, 18, 294-300. 
24. Dawood, K.M.; Farag, A.M.; Abdel-Aziz, H.A. Synthesis and antimicrobial evaluation of some 1,2,4-triazole, 1,3,4-oxa(thia)diazole and 1,2,4-triazolo[3,4-b]-1,3,4-thiadiazine derivatives. Heteroatom Chem. 2005, 16, 621-627.

25. Bhalla, M.; Shukla, S.; Gujrati, V.R.; Saxena, A.K.; Sanger, K.C.; Shaker, K. Anti-inflammatory benzopyran-2-ones and their active oxygen species (aos) scavenging activity. Boll. Chim. Farm. 1998, 137, 403-411.

26. Bhat, M.A.; Siddiqui, N.; Khan, S.A. Synthesis of novel 3-(4-acetyl-5- $H /$ methyl-5-substituted phenyl-4,5-dihydro-1,3,4-oxadiazol-2-yl)-2H-chromen-2-ones as potential anticonvulsant agents. Acta Pol. Pharm. Drug Res. 2008, 65, 235-239.

27. Bhalla, M.; Hitkari, A.; Gujrati, V.R.; Bhalla, T.N.; Shanker, K. Benzopyran-2-one derivatives: Antiinflammatory, analgesic and antiproteolytic agents. Eur. J. Med. Chem. 1994, 29, 713-717.

28. Raslan, M.A.; Khalil, M.A. Heterocyclic synthesis containing bridgehead nitrogen atom: Synthesis of 3-[(2H)-2-oxobenzo[b]pyran-3-yl]-s-triazolo[3,4-b]-1,3,4-thiadiazine and thiazole derivatives. Heteroatom Chem. 2003, 14, 114-120.

29. Khan, M.S.Y.; Akhtar, M. Synthesis of some new 2,5-disubstituted 1,3,4-oxadiazole derivatives and their biological activity. Indian J. Chem. 2003, 42B, 900-904.

30. RamaGanesh, C.K.; Bodke, Y.D.; Venkatesh, K.B. Synthesis and biological evaluation of some innovative coumarin derivatives containing thiazolidin-4-one ring. Indian J. Chem. 2010, 49B, 1151-1154.

31. Singh, V.; Srivastava, V.K.; Palit, G.; Shanker, K. Coumarin congeners as antidepressants. Arzneim-Forsch. Drug Res. 1992, 42, 993-996.

32. Sivakumar, K.K.; Rajasekaran, A.; Ponnilavarasan, I.; Somasundaram, A.; Sivasakthi, R.; Kamalaveni, S. Synthesis and evaluation of anti-microbial and analgesic activity of some (4Z)-3methyl-1-[(2-oxo-2H-chromen-4-yl)carbonyl]-1H-pyrazole-4,5-dione-4-[(4-substitutedphenyl)hydrazone]. Der Pharmacia Lett. 2010, 2, 211-219.

33. Cardoso, S.H.; Barreto, M.B.; Lourenço, M.C.S.; das Graças, M.; Henriques, M.D.O.; Candéa, A.L.P.; Kaiser, C.R.; de Souza, M.V.N. Antitubercular activity of new coumarins. Chem. Biol. Drug Des. 2011, 77, 489-493.

34. Patil, S.A.; Unki, S.N.; Kulkarni, A.D.; Naik, V.H.; Badami, P.S. Synthesis, characterization, in vitro antimicrobial and DNA cleavage studies of $\mathrm{Co}(\mathrm{II}), \mathrm{Ni}(\mathrm{II})$ and $\mathrm{Cu}(\mathrm{II})$ complexes with ONOO donor coumarin Schiff bases. J. Mol. Struct. 2011, 985, 330-338.

35. Sreeja, S.; Mathan, S.; Kumaran J. Design, synthesis and pharmacological evaluation of new coumarin derivatives. Int. J. Adv. Pharm. Biol. Sci. 2012, 2, 80-91.

36. Biradar, J.S.; Manjunath, S.Y. Synthesis of novel 2-(5'-substituted-2'-phenylindole-3'-yl)-5(coumarin-3"-yl)-1,3,4-oxadiazoles and 4-(5'-substituted-2'-phenylindole-3'-yl)-1-(coumarin-3"amido)azetidin-2-one and their antimicrobial activity. Indian J. Chem. 2004, 43B, 141-143.

37. Islam, A.M.; Bedair, A.H.; Aly, F.M.; El-Sharief, A.M.Sh.; El-Masry, F.M. Synthesis and reactions of coumarin-3- $N$-bromo-arylcarboxamides. Indian J. Chem. 1980, 19B, 224-227.

38. Soliman, F.S.G.; Labouta, I.M.; Stadlbauer, W. Reactions of some coumarins with hydrazine and phenyl hydrazine. Arch. Pharm. Chim. 1985, 13, 49-52.

39. Li, D.; Tan, M.X.; Jie, L. Synthesis, antioxidant and antibacterial activities of salicylaldehyde azine. Adv. Mat. Res. 2012, 396-398, 2366-2369. 
40. Metwally, S.A.M.; AbdelMoneim, M.I.; Elossely, Y.A.; Awad, R.I.; Abou-Hadeed, K. Synthesis and crystal structure of some 3,5-pyrazolidinediones. Chem. Het. Comp. 2010, 46, 426-437.

41. Rozin, Yu.A.; Vorob'ova, E.A.; Morzherin, Yu.Yu.; Bakulev, V.A. Synthesis and investigation of ring-chain isomerism of the derivatives of $\mathrm{N}$-amino-5-hydroxy-1,2,3-triazole -4-carboxylic acid. Chem. Het. Comp. 2001, 37, 294-304.

Sample Availability: Samples of the compounds are available from Dr. Hatem A. Abdel-Aziz, Department of Pharmaceutical Chemistry, College of Pharmacy, King Saud University, P.O. Box 2457, Riyadh 11451, Saudi Arabia.

(C) 2013 by the authors; licensee MDPI, Basel, Switzerland. This article is an open access article distributed under the terms and conditions of the Creative Commons Attribution license (http://creativecommons.org/licenses/by/3.0/). 